\title{
A New Method in Pilot Reuse Factor Selection in Spectrum Efficient Massive MIMO Systems
}

\author{
Osman Dikmen*, Selman Kulac \\ Department of Electrical Electronics Engineering, Faculty of Engineering, Duzce University, \\ Duzce, Turkey \\ osmandikmen@duzce.edu.tr
}

\begin{abstract}
Spectrum efficiency studies of Massive MIMO systems have continued and still have not been fully explored in the literature. Therefore, the spectrum efficiency definition of a particular user in a cell in a Massive MIMO network is expressed in this paper. In addition, the uplink spectrum efficiency expressions for the Rayleigh fading are included. The Monte Carlo simulations are performed to verify these expressions. Due to the expectation of reaching to a thousand antennas together with the millimetre wave structure in the next years, in this study, it is contributed to the literature by explaining how to select the pilot reuse factor. The realization of this contribution is based on Zero Forcing (ZF) and Maximum Ratio Combining (MRC) schemes. The results of this study are useful for optimal design conditions, such as the number of antennas on the base station and pilot reuse factor selection for the next generation networks in order to obtain spectrum efficiency in Massive MIMO systems.
\end{abstract}

Index Terms-Massive MIMO; Millimetre wave; Pilot reuse factor; Spectrum efficiency.

\section{INTRODUCTION}

Achieving spectrum efficiency is an important priority for next generation wireless communications technologies. Because one of the problems in wireless communications technologies is crowded electromagnetic spectrum. Although wireless throughput is increasing day by day, the electromagnetic spectrum will never increase [1]. Achieving the spectrum efficiency is an important issue, especially for 5 th generation $(5 \mathrm{G})$ wireless networks. $5 \mathrm{G}$ communication systems require improvements, such as higher data rate/spectrum efficiency, lower latency, and more environmentally friendly, which is called "green communication", compared to 4th generation (4G) [2]. One of the ways to achieve spectrum efficiency is to use very large multiple input multiple output (Massive MIMO) systems.

Massive MIMO, which has recently been employed in wireless communications technologies, is known as a system, in which communication is performed by multiple antennas in the receiver and transmitter. Massive MIMO has been variously called in literature, such as "very large MIMO", "Large Scale Antenna Systems", etc. [3]. But the standards of Massive MIMO have not been fully explained

Manuscript received 16 January, 2019; accepted 23 May, 2019.

This paper was supported by the Scientific Research Project Coordination Unit of Duzce University (Project No. 2017.06.03.530). yet. However, several studies have been carried out using hundreds of antennas in the base station (BS) recently. The main benefits of using more than one antenna are higher performance through diversity and higher data rates through spatial multiplexing. There is no limit to the number of antennas.

In Massive MIMO, various benefits can be realized with the use of hundreds of antennas as cylindrical, linear, and rectangular forms in BS. Some of these benefits are briefly as follows. The capacity can be increased 10 times with Massive MIMO. At the same time, energy efficiency is achieved also [4]. The large number of antennas in BS enhances the robustness of the system against fading and various impairments [5]. It is also possible to reduce latency due to the communication between large number of users with BSs, which have tens of antennas. In addition, it is presented that the system is more robust against the interference in this way.

The advantages of Massive MIMO are important in the development of $5 \mathrm{G}$ and beyond wireless networks. When the recent studies are examined, there are many studies on the above-mentioned improvements. In one of these studies [6], the theoretical work on network design, signal processing, and signal coding for the Massive MIMO system is presented. In another study, a method for deriving the concepts for high mobility and low signal to noise ratio (SNR) conditions caused by multi-user MIMO is introduced [7]. In addition, the benefits of a HetNet system have been mentioned which is combined Massive MIMO with mmWave [8]. It is also studied to determine how many antennas are needed for Massive MIMO in the uplink and downlink cellular networks [9].

So, there are many studies dedicated for the development of $5 \mathrm{G}$ and beyond wireless networks and many theoretical analyses are presented in the literature also. In this study, it is aimed to realize the spectrum efficiency which has a very important pointin next generation wireless networks. Because of the range of bandwidth that can be processed is limited, the spectrum efficiency needs to be increased. Moreover, the use of the efficient spectrum will become even more important as the number of Internet of Things devices increases [10]. The frequency bands of current conventional systems are below $6 \mathrm{GHz}$. However, since these frequency bands are used extensively, they will not be sufficient for 5G and beyond communication [11]. 
Therefore, it is required to ensure spectrum efficiency. To this end, the Massive MIMO system is considered. The uplink and downlink of the Massive MIMO system [1] are indicated in Fig. 1(a) and Fig. 1(b), respectively.

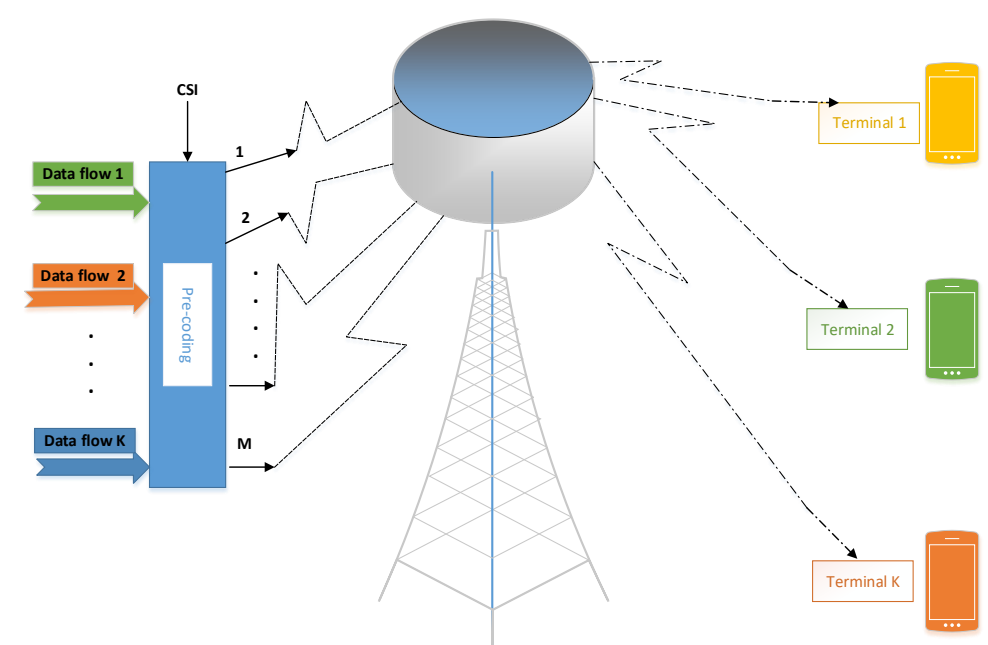

(a)

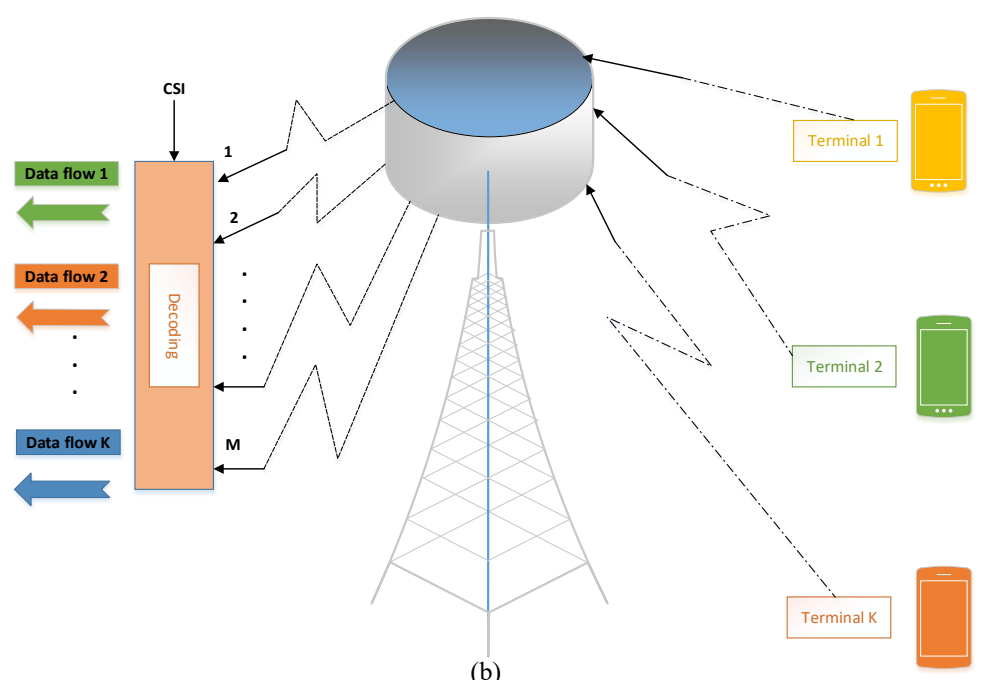

Fig. 1. Massive MIMO (BS with M antennas): (a) Downlink; (b) Uplink.

Massive MIMO theoretical analyses are quite extensive for the mentioned improvements in the literature. In this study, instead of these full theoretical analyses in the literature, only parts for spectrum efficiency analysis are considered. Thus, the spectrum efficiency of the Massive MIMO system, which will be used in 5G and beyond wireless networks, is analysed. The summary of the contribution of this study is as follows:

1. The lower bounds on the total capacity are specified for the downlink with linear pre-coding, for uplink - with linear detection. Thanks to this detailed mathematical modelling, spectrum efficiencies are shown in the perfect Channel State Information (CSI) for a fixed number of users and different BS antenna numbers.

2. The Time Division Duplexing (TDD) system is also considered and emphasized by the help of the literature, i.e., that the spectrum efficiency significantly increases with addition number of BS antennas for the fixed users with several SNR values.

3. In general, through the lemmas in the literature, the equations of achievable rates are tried to be expressed in SNR regions. Thus, spectrum efficiency for Massive MIMO is determined by mutual information. SNR values are required to determine this mutual information Researchers are encouraged to express these equations clearly.

4. Finally, it is highlighted how the pilot reuse factor should be selected when number of BS antennas reaches to thousand, what is expected in future generation wireless networks. Thereby, the spectrum efficiency is maximized by determining the number of antennas based on the selection of the pilot reuse factor.

The remaining of the paper is organized as follows. Section II shows the system model of Massive MIMO, which is generally used in the literature for spectrum efficiency. In Section III, literature analyses of spectrum efficiency for Massive MIMO systems are given. In Section IV, numerical analysis results of spectrum efficiency expressions, which are obtained from literature lemmas, are shown using Monte Carlo simulation. Finally, the conclusions take place in Section V.

The representations used in the paper are as follows. Bold lowercase and uppercase letters are used to represent vectors and matrices, respectively. Transpose, conjugate transpose, absolute function, and expected value are expressed by $(.)^{\mathrm{T}}$, $(.)^{\mathrm{H}}$, I.|, and $\mathrm{E}[$.$] , respectively. \mathrm{C}^{m x n}$ indicates an $m x n$ complex 
matrix.

\section{SYSTEM MODEL}

In this Section, the Massive MIMO system used in the literature for spectrum efficiency is presented. Here, a multicell structure is mentioned, where the number of BS antennas and the number of users are expressed by $\mathrm{M}$ and $\mathrm{K}$, respectively. The channel model of the system is Rayleigh fading and it is considered to serve in TDD mode. The basic function of the Massive MIMO system is that as the number of BS antennas increases the correlation between different User Equipment (UE) is less [6]. In case of Rayleigh fading [12], when number of BS antennas is significantly large, the channel between various users of the same cell becomes asymptotically orthogonal. Figure 2 represents a model of MIMO transmission.

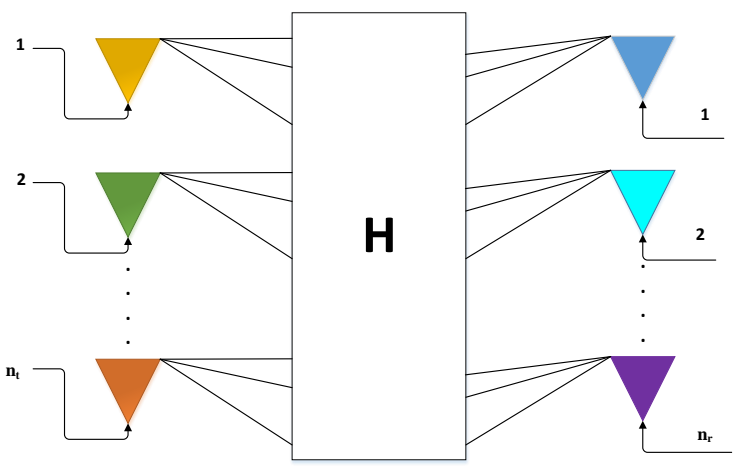

Fig. 2. MIMO transmission in $\mathrm{H}$ channel.

If there are $n_{t}$ transmitter and $n_{r}$ receiver antennas in the system, the signals obtained at the receiver can be written as follows

$$
\mathbf{y}=H \mathbf{x}+\mathbf{v},
$$

where $\mathbf{y}$ is the received signal vector $\left(\mathrm{n}_{\mathrm{r}} \times 1\right), \mathbf{x}$ is the transmitted signal vector $\left(n_{t} \times 1\right), \mathbf{H}$ is the channel matrix $\left(\mathrm{n}_{\mathrm{r}} \times \mathrm{n}_{\mathrm{t}}\right)$, and $\mathbf{v}$ represents noise vector $\left(\mathrm{n}_{\mathrm{r}} \times 1\right)$ at the receiver.

We can determine (2) using (1) as follows

$$
\frac{h_{n}^{H}}{n_{r}}=\frac{h_{n}^{H} h_{n} x_{n}}{n_{r}}+\sum_{i \neq n}^{n_{i}} \frac{h_{n}^{H} h_{i} x_{i}}{n_{r}}+\frac{h_{n}^{H} v}{n_{r}},
$$

where $h_{i}$ is stated ith channel vector between the transmitter and the receiver [13].

\section{A. Massive MIMO System}

A massive MIMO network, which has $\mathrm{K}$ single antenna users and BS with $\mathrm{M}$ antennas in L cells, is considered. In this system, the channel feedback is denoted by $\mathbf{h}_{i, k}^{\prime}=\left[h_{i, k, 1}^{l} \ldots h_{i, k, M}^{l}\right]^{T} \in C^{M}$,

where $1, \mathrm{k}$ and I stands for BS, user, and cell, respectively. The variance of mth coefficient of $h_{i, k}^{l}$ is formulated as follows

$$
\beta_{i, k}^{l}=V\left\{h_{i, k, m}^{l}\right\}
$$

Then, using these channel properties, the uplink and downlink process is shown [13].

\section{B. Uplink with Linear Detection}

The baseband signal $y_{l} \in C^{M}$ obtained from lth BS is modeled for each uplink symbol as follows using the equations in [14]

$$
y_{l}=\sum_{i=1}^{L} \sum_{k=1}^{K} \mathbf{h}_{i, k}^{l} \sqrt{P_{i, k}} x_{i, k}+\mathbf{n}_{l},
$$

where $x_{\mathrm{i}, \mathrm{k}}$ is the normalized transmittal symbol and $P_{\mathrm{i}, \mathrm{k}}$ is the transmit power of the user $\mathrm{k}$ in cell. By means of $H_{i}^{l}=\left\lfloor h_{i, 1}^{l} \ldots h_{i, K}^{\prime}\right\rfloor \in C^{M X K}, \quad \mathbf{P}_{i}=\operatorname{diag}\left(p_{i, 1} \ldots p_{i, K}\right) \in C^{K X K}, \quad$ and $x_{i}=\left[x_{i, 1} \cdots x_{i, K}\right]^{T} \in C^{K}$ matrix representations, (5) is obtained

$$
y_{l}=\sum_{i=1}^{L} H_{i}^{l} P_{i}^{1 / 2} x_{i}+n_{i}
$$

The overall scheme of uplink transmission is indicated in Fig. 3.

The process taking place in the diagram shown in Fig. 3 is to try to equalize the $\tilde{x}_{l, k}$ signal, which is determined at BS 1 to the real signal $x_{l, k}$.

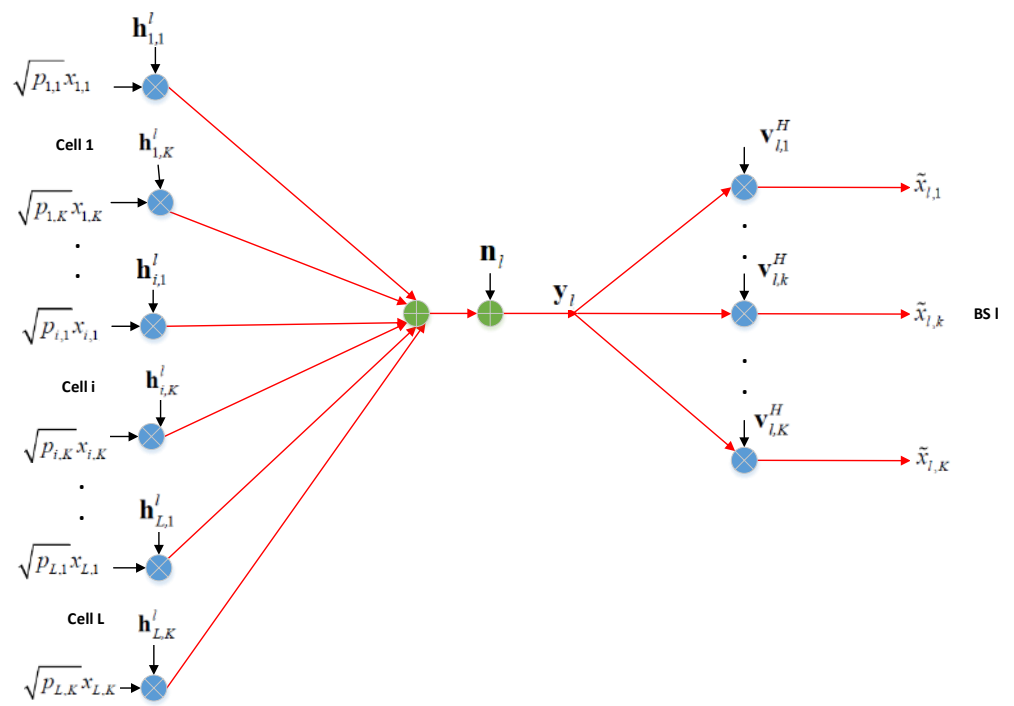

Fig. 3. Uplink transmission scheme in a Massive MIMO system. 
In uplink transmission, BS in the lth cell only detects its own signals using the $y_{l}$ signal indicated in (4). The interference of the transmitted signal, which occurs during transmission, is eliminated by the $v_{l, k} \in C^{M}$ linear detection vector. This is accomplished by the equations in [15] and [16] as follows

$$
\begin{aligned}
& v_{l, k}^{H}=\sum_{i=1}^{L} \sum_{t=1}^{K} v_{l, k}^{H} h_{l, k}^{l} \sqrt{P_{i, t}} x_{i, t}+v_{l, k}^{H} n_{l}= \\
& =v_{l, k}^{H} h_{l, k}^{l} \sqrt{P_{l, k}} x_{l, k}+\sum_{t=1}^{K} v_{l, k}^{H} h_{l, t}^{l} \sqrt{P_{l, t}} x_{l, t}+ \\
& +\sum_{i=1}^{L} \sum_{t=1}^{K} v_{l, k}^{H} h_{i, t}^{l} \sqrt{P_{l, t}} x_{i, t}+v_{l, k}^{H} n_{l,},
\end{aligned}
$$

where $x_{i, t}$ is the data symbol, which is transmitted by the user $\mathrm{t}$ in cell $\mathrm{i}$.

\section{Downlink with Linear Pre-Coding}

Downlink in the Massive MIMO system, the signal vector sent for user $\mathrm{k}$ in BS 1, is denoted by (7) [14]

$$
x_{l}=\sum_{i=1}^{L} \sqrt{P_{l, t}} w_{l, l} S_{l, t}
$$

It is assumed that $S_{l, t}$ is the payload symbol $P_{l, t}$ represents the transmit power. If $\mathrm{t}=1,2,3 \ldots \mathrm{K}, \mathbf{w}_{l, t}$ is the linear precoding vector. The signal obtained for $\mathrm{k}$ users in cell 1 is derived as (8)

$$
y_{l, k}=\sum_{i=1}^{L}\left(\mathbf{h}_{l, k}^{i}\right)^{H} \mathbf{x}_{i}{ }^{+} n_{l, k}
$$

Downlink transmission model is given in Fig. 4.

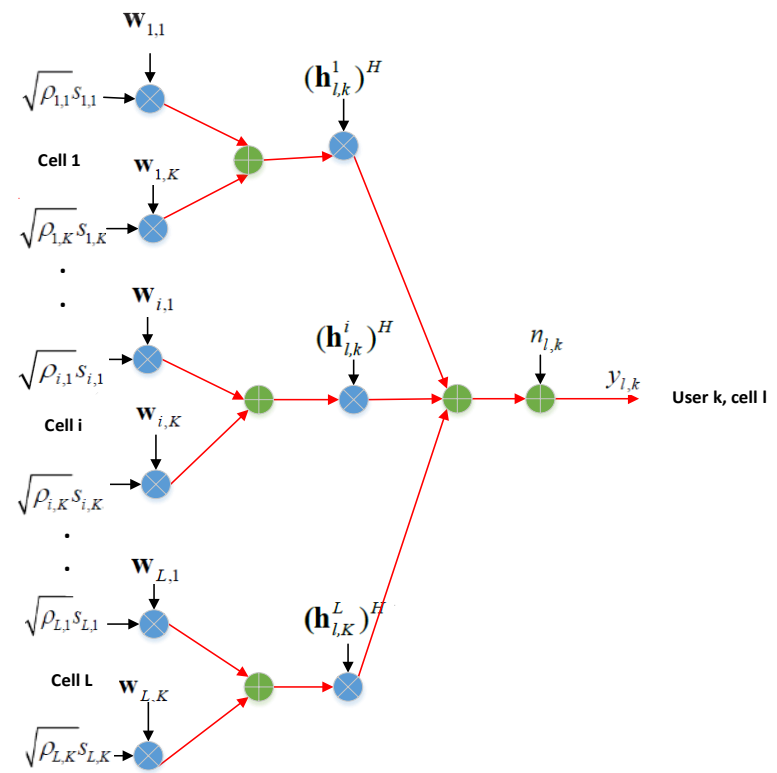

Fig. 4. Downlink transmission plan in multi-cell MIMO system.

\section{SPECTRUM EFFICIENCY ANALYSIS OF MASSIVE MIMO}

There are many analyses in the literature to examine various performances of Massive MIMO systems. An important contribution of this study is that, instead of the complexity of the analysis in the literature, only spectrum efficiency expressions in Massive MIMO are clearly shown. To accomplish this, five literature lemmas are presented.

\section{A. Literature Lemma 1}

The equations, which are needed to obtain the channel estimate $\hat{\mathbf{h}}_{i, k}^{\prime}$ of the real channel $\mathbf{h}_{i, k}^{\prime}$, are summarized by reviewing the literature. Linear Minimum Mean Square Error (LMMSE) for channel estimation, Zero Forcing (ZF), and Maximum Ratio Combining (MRC) schemes for detection are considered.

Using the LMMSE estimation definition in [17], the channel estimate for $h_{j, k, m}^{l}$ is shown as follows

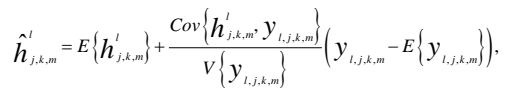

where $y_{l, j, k, m}$ is specified as follows [13]

$$
y_{l, j, k, m}=\sum_{i \in \mathfrak{R},} \sqrt{p_{i, k}} \tau_{p} h_{i, k, m}^{l}+\tilde{n}_{l, j, k, m}
$$

here $E\left\{h_{j, k, m}^{\prime}\right\}=\bar{h}_{j, k, m}^{l}, \operatorname{Cov}\{\ldots\}$, and $v$ represent the mean value vector, covariance, and variance, respectively. These can be written as [13]:

$$
\begin{gathered}
E\left\{y_{l, j, k, m}\right\}=\sum_{i \in \Re_{j}} \sqrt{p_{i, k}} \tau_{p} \bar{h}_{i, k, m}^{\prime}, \\
\operatorname{Cov}\left\{h_{j, k, m}^{l}, y_{l, j, k, m}\right\}=\sqrt{p_{j, k}} \tau_{p} \beta_{j, k}^{l}, \\
V\left\{y_{l, j, k, m}\right\}=\sum_{i \in \mathfrak{R}_{j}} p_{j, k} \tau_{p}^{2} \beta_{j, k}^{l}+\tau_{p} \sigma_{U L}^{2} .
\end{gathered}
$$

In summary, in Literature lemma 1, the equations that can be used for bit error rates and channel estimation in Massive MIMO networks are shown. Based on the above propagation assumptions, in section IV, it is shown that the efficiency of the Massive MIMO system can be obtained according to different number of antennas in the BS together with the precoding.

\section{B. Literature Lemma 2}

Here, the definition of $S N R_{l, k}^{U L}$, which is related with the available spectrum efficiency of $\mathrm{k}$ users in cell 1 in the Massive MIMO network, is shown. In the uplink, the lower bound on the ergodic capacity of $\mathrm{k}$ user in cell 1 is given as follows [13]

$$
R_{l, k}^{v L}=\gamma^{u L}\left(1-\frac{\tau_{p}}{\tau_{c}}\right) \log _{2}\left(1+S N R_{l, k}^{v L}\right) .
$$

The SNR is expressed as follows [13]

$$
S N R_{l, k}^{u L}=\frac{p_{l, k}\left|E\left\{\mathbf{v}_{l, k}^{H} \mathbf{h}_{l, k}^{l}\right\}\right|^{2}}{\sum_{i=1}^{L} \sum_{l=1}^{K} p_{i, t} E\left\{\left|\mathbf{v}_{l, k}^{H} \mathbf{h}_{l, k}^{l}\right|^{2}\right\}-p_{l, k}\left|E\left\{\mathbf{v}_{l, k}^{H} \mathbf{h}_{l, k}^{l}\right\}\right|^{2}+\sigma_{v L}^{2} E\left\{\left\|\mathbf{v}_{l, k}\right\|^{2}\right\}}
$$

\section{Literature Lemma 3}

The expressions to be used in MRC and ZF detection are shown for spectrum efficiency. The MRC detection is intended to maximize the numerator of $S N R_{t, k}^{U L}$ expression, while the ZF detection aims to reduce interference from intercellular.

First, in MRC detection, when $v_{l, k}=\hat{\mathbf{h}}_{l, k}^{\prime} S N R_{l, k}^{u L}$ in Literature lemma 2 is as follows [18] 


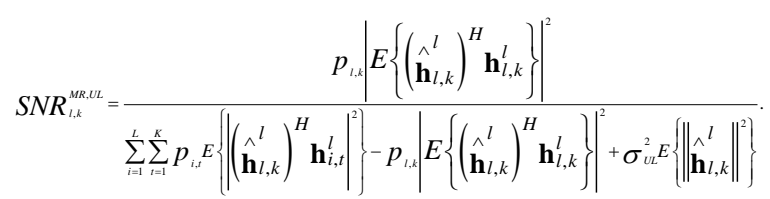

For ZF detection, $E\left\{v_{l, k}^{l}\right\}=1$. In addition, the complex Wishart matrix in (15) is stated as follows [19]

$$
\left.\sigma_{v L}^{2} E\left\{\left\|\mathbf{v}_{l, k}\right\|^{2}\right\}=\sigma_{u L}^{2} E\left\{t\left[\left(\hat{\mathbf{H}}_{l}^{l}\right)^{H} \hat{\mathbf{H}}_{l}^{l^{-1}}\right]_{k, k}\right\}=\frac{\sigma_{u L}^{2}}{(M-K) V\left\{\left\{\hat{h}_{l, k, m}^{\prime}\right.\right.}\right\} .
$$

Placing $E\left\{v_{l, k}^{l}\right\}=1$ and (17) in (15), ZF is obtained as follows [18]

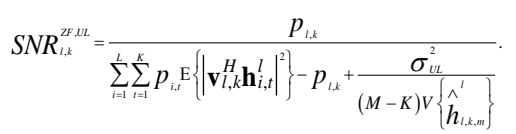

\section{Literature Lemma 4}

This section gives SNR expression for the lower bound and spectrum efficiency. These expression was used in simulation for downlink way. In downlink, lower bound of the ergodic rate for user $\mathrm{k}$ in cell 1 is given as follows [13]

$$
R_{l, k}^{D L}=\gamma^{D L}\left(1-\frac{\tau_{p}}{\tau_{c}}\right) \log _{2}\left(1+S N R_{l, k}^{D L}\right)
$$

where the SNR is expressed as follows

$$
S N R_{l, k}^{D L}=\frac{p_{l, k} \mid \mathrm{E}\left\{\left.\begin{array}{ll}
h_{l, k}^{l}{ }^{H} & \left.w_{l, k}\right\}
\end{array}\right|^{2}\right.}{\sum_{i=1}^{L} \sum_{t=1}^{K} p_{i, t} \mathrm{E}\left\{\left|h_{l, k}^{l}{ }^{H} w_{i, t}\right|^{2}\right\}-\sum_{t=1}^{K} p_{i, t} \mathrm{E}\left\{\left|h_{l, k}^{l}{ }^{H} w_{i, t}\right|^{2}\right\}} .
$$

\section{E. Literature Lemma 5}

The pre-coding vector required for downlink is stated with the help of studies in [18] and [20].

The downlink pre-coding vectors are denoted as follows subject to uplink detection vectors $v_{l, k}$ for all 1 and $\mathrm{k}$

$$
\mathbf{w}_{l, t}=\frac{\mathbf{v}_{l, k}}{\sqrt{E\left\{\left\|\mathbf{v}_{l, k}\right\|^{2}\right\}}} .
$$

That is, the channel estimate for uplink is also used for the downlink linear pre-coding. Five lemmas in literature are used for the numerical analysis results.

\section{NUMERICAL ANALYSIS RESULTS}

Simulation results are presented to demonstrate the accuracy of analyses given by lemmas in literature for $5 \mathrm{G}$ and beyond networks and to examine the spectrum efficiency gains of Massive MIMO system with different parameters. These results are shown in 3 steps as follows.

\section{A. Spectrum Efficiency in Massive MIMO System with Different Number of BS Antennas and Fixed Number of User}

Spectrum efficiency performances are obtained for Massive MIMO systems for each user. The parameters used for this simulation are shown in Table I under the assumption that perfect CSI and channels are modeled by Rayleigh fading.

TABLE I. SIMULATION PARAMETERS.

\begin{tabular}{|c|c|}
\hline System service & Value \\
\hline Average SNR of user $(\mathrm{dB})$ & $0,5,10,20$ \\
\hline Number of BS antennas, $\mathrm{M}$ & From 0 to 100 \\
\hline Number of user, $\mathrm{K}$ & 10 \\
\hline
\end{tabular}

The ZF scheme is considered for Fig. 5, where the results are for different SNR values and the average total spectrum efficiency values are shown as a function of $\mathrm{M}$. The results were performed for both uplink and downlink. In addition, Monte Carlo simulation was used to realize the results.

In simulation, it is seen that the capacity increases when $M>K$ and the ZF performance approaches the theoretically considered capacity (there is not any interference). Even when $M \gg r$, the spectrum efficiency gain is very close to the theoretical gain.

\section{B. Determination of Pilot Reuse Factor for Spectrum Efficiency in Massive MIMO System}

Here, the effect of the pilot reuse factor on the spectrum efficiency is analysed. It is clear that BSs will be equipped with thousands of antennas in next generation wireless networks with Massive MIMO. In such a case, this simulation is carried out to show how the selection of the pilot reuse factor should be. The results are obtained for both MRC and ZF detection and the parameters used in the simulation are shown in Table II.

TABLE II. SIMULATION PARAMETERS.

\begin{tabular}{|c|c|}
\hline System service & Value \\
\hline Average SNR of user $(\mathrm{dB})$ & 5 \\
\hline Coherence block size $\left(\boldsymbol{\tau}_{\mathrm{E}}\right)$ & 400 \\
\hline Number of BS antennas, $\mathrm{M}$ & From 0 to 1000 \\
\hline Number of user, $\mathrm{K}$ & 100 \\
\hline Path loss coefficient & 4 \\
\hline
\end{tabular}

The pilot reuse factor $f=\tau_{p} / K$ is an integer, where $\mathrm{K}$ is the number of users per cell. $\mathrm{f}=1$ is called as universal pilot reuse, and $f>1$ is named as non-universal pilot reuse factor [21]. When $\mathrm{f}=1$, inter-cell interference is caused during the channel estimation, which can be eliminated by using different pilot reuse factors [22].

If the BS antenna number is close to thousand, the numerical analysis is performed for assuming pilot reuse factors $f \in\{1,3,4,7\}$,.ig. 6 shows the average spectrum efficiency for MR detection and Fig. 7 shows the average spectrum efficiency for $\mathrm{ZF}$ detection as function of pilot reuse factors.

From the results, it is observed that $f=3$ pilot reuse is better when low load, i.e., number of antennas in BS is not large. When $f$ is selected appropriately, it can work on the upper side of a curve in Fig. 6 and Fig. 7 and, thus, Massive MIMO can provide high spectrum efficiency with a thousand antennas in BS. 


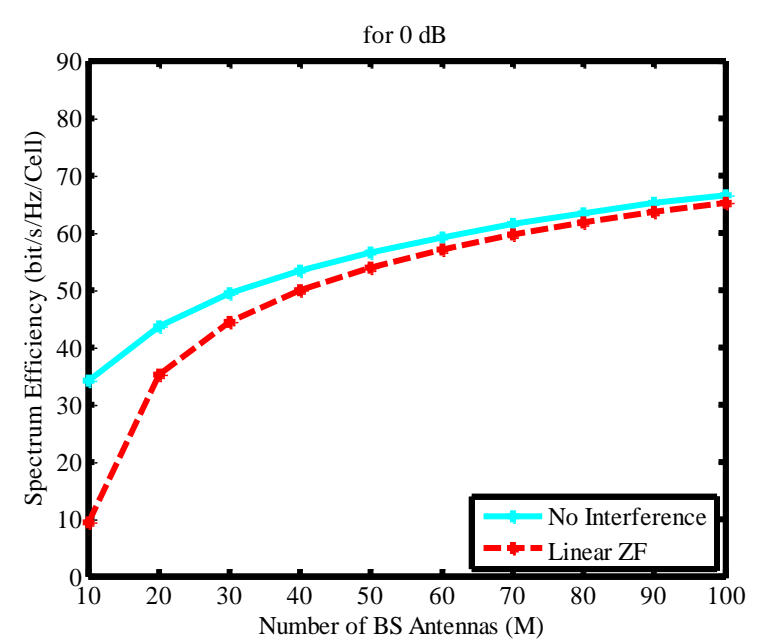

(a)

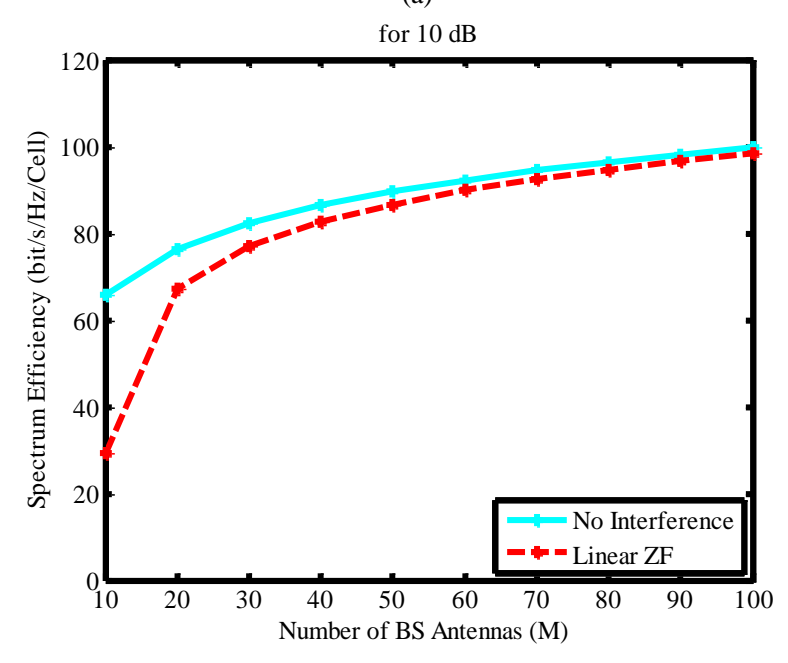

(c)

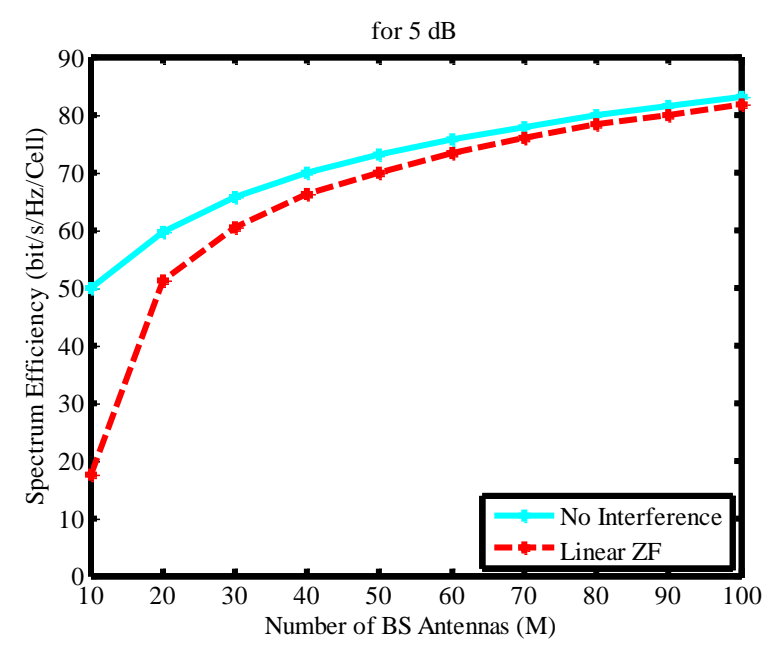

(b)

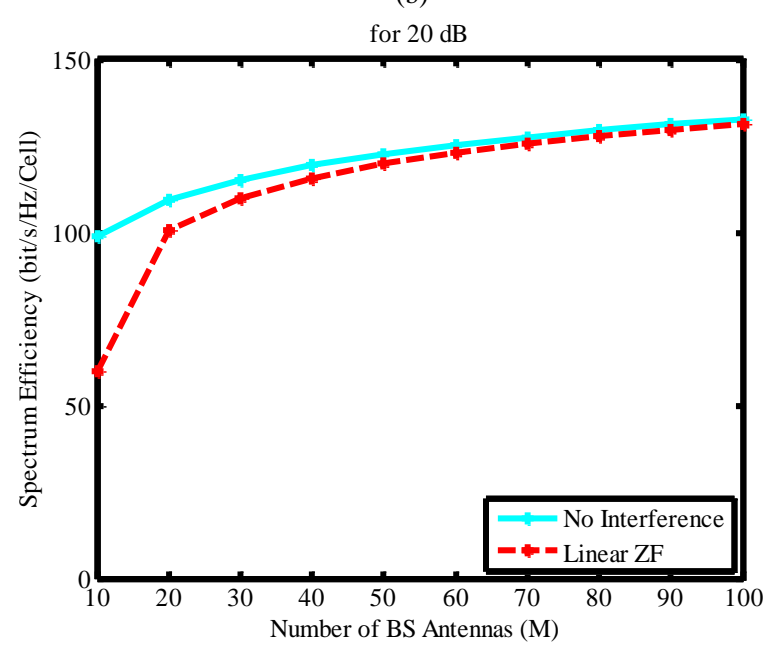

(d)

Fig. 5. Spectrum efficiency in massive MIMO system with different number of BS antennas and constant number of user: (a) for 0dB; (b) for $5 \mathrm{~dB}$; (c) for $10 \mathrm{~dB}$; (d) for $20 \mathrm{~dB}$.

Moreover, in MRC, it is observed that the pilot reuse factor 3 is more suitable for the number of antennas in the base station is about a hundred as in the literature. However, unlike in the literature, it is observed that the pilot reuse factor should be selected a more appropriate value when considering the number of more antennas in future technologies. When the system with MRC is examined, the selecting pilot reuse factor 3 around a hundred antennas is important to increase the spectrum efficiency. But, when the number of antennas is about 200 and above, it is considered that selecting reuse factor 1 is ideal.

A similar situation is seen in ZF detection. It is understood that the pilot reuse factor used when the number of antennas is a hundred or less needs to be changed in order to increase spectrum efficiency in the system, where the number of antennas increases to about a thousand. In ZF, it is important to select pilot reuse factor 1 when number of BS antennas is above 600. Similarly, using pilot reuse factor 3 is very efficiency, while the number of BS antennas is up to about 600. It is possible to state, which pilot reuse factor is used for which antenna number ranges using the results obtained. These results make it easy to determine the design conditions also.

Another observation to be obtained as a result of the numerical analysis, is that the spectrum efficiency between ZF and MRC (Fig. 6 and Fig. 7) is the different. These results show that ZF is better than MRC. Briefly, the pilot reuse factor is a significant ratio in Massive MIMO systems, and the most suitable selection rely on the number of BS antennas.

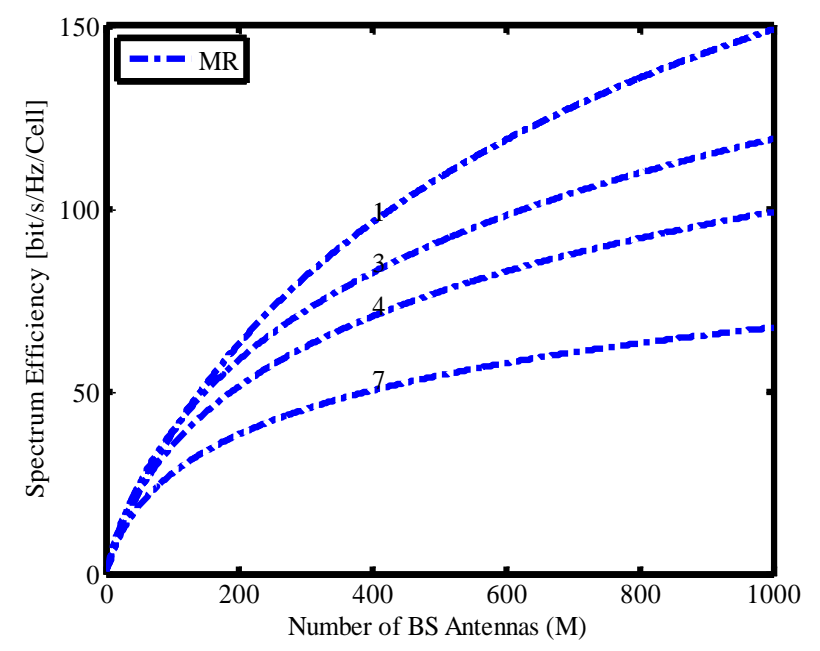

Fig. 6. Spectrum efficiency in different pilot reuse factors for MR detection. 


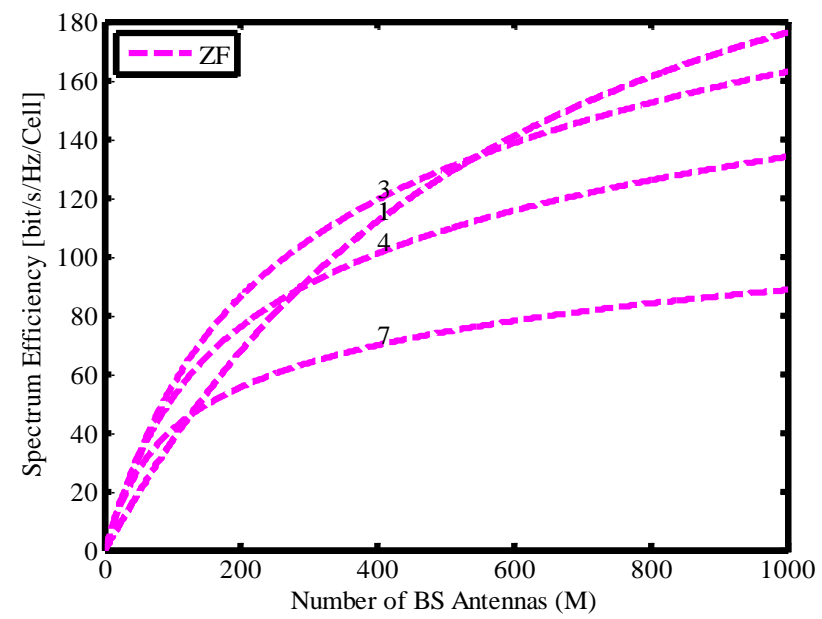

Fig. 7. Spectrum efficiency in different pilot reuse factors for ZF detection.

\section{Spectrum Efficiency with Massive MIMO in $5 G$ Networks}

The contributions of Massive MIMO systems for the spectrum efficiency are tried to be shown in the above results. In this section, it is shown the spectrum efficiency performance that will be achieved with Massive MIMO uplink and downlink of $5 \mathrm{G}$ networks. The pilot reuse factor is set to $f=3 \mathrm{ZF}$ detection is considered and $5 \mathrm{~dB}$ SNR is assumed to each user.

Figure 8 shows the spectrum efficiency as a function of the BS antenna number M. Also, the maximum number of users per cell is 10 . The performance of these results is compared to 4G networks [2]. When compared with International Mobile Telecommunications-Advanced (IMTAdvanced), 39bit/s/ Hz/cell is obtained for $M=100$ antennas and an increase of 15 to 25 times in spectrum efficiency is observed. In $M=400$ antennas massive MIMO system, the spectrum efficiency is $57 \mathrm{bit} / \mathrm{s} / \mathrm{Hz} / \mathrm{cell}$. This improvement provides from 35 to 50 times more gain than IMT-Advanced.

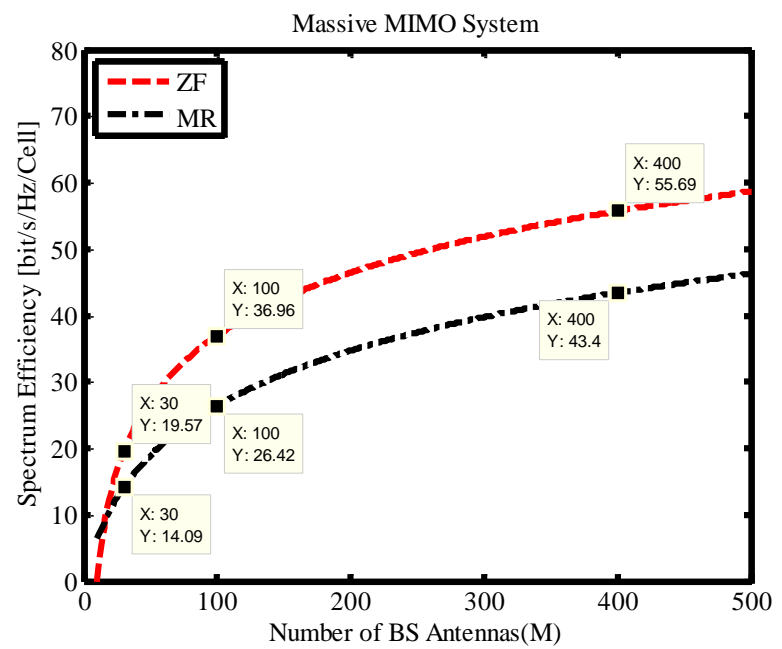

Fig. 8. Spectrum efficiency in pilot reuse factor $\mathrm{f}=3$ and $5 \mathrm{~dB}$ SNR depending on BS antenna for $\mathrm{ZF}$ and MR detection.

\section{V.CONCLUSIONS}

In this study, the spectrum efficiency performance of Massive MIMO to be used in next generation wireless networks is analysed. For this purpose, the theoretical analyses in the literature are examined and only the output obtained at the point of spectrum efficiency is presented. Thus, numerical analyses for spectrum efficiency, which is a special field, are carried out for Massive MIMO, instead of general concepts in the literature. As a result, if the number of $\mathrm{M}$ antennas is much larger than the number of $\mathrm{K}$ users, it is shown that the theoretical results are approached. It is also emphasized that the pilot reuse factor is a significant index in Massive MIMO systems and should be selected according to the number of antennas. Finally, it is observed that spectrum efficiency improvements of ten times or more are achieved according to the IMT-Advanced, which is theoretical results presented for $4 \mathrm{G}$ systems.

\section{CONFLICTS OF INTEREST}

The authors declare that they have no conflicts of interest.

\section{REFERENCES}

[1] T. L. Marzetta, "Massive MIMO: An introduction", Bell Labs Tech. J., vol. 20, pp. 11-22, Mar. 2015. DOI: 10.15325/BLTJ.2015.2407793.

[2] J. G. Andrews, S. Buzzi, W. Choi, S. V. Hanly, A. Lozano, A. C. K. Soong, and J. C. Zhang, "What will 5G be?", IEEE J. Sel. Areas Commun., vol. 32, no. 6, pp. 1065-1082, Jun. 2014. DOI: 10.1109/JSAC.2014.2328098.

[3] E. Larsson, O. Edfors, F. Tufvesson, and T. Marzetta, "Massive MIMO for next generation wireless systems", IEEE Commun. Mag., vol. 52, no. 2, pp. 186-195, Feb. 2014. DOI: 10.1109/MCOM.2014.6736761.

[4] Z. Ding, R. Schober, and H. V. Poor, "A general MIMO framework for NOMA downlink and uplink transmission based on signal alignment", IEEE Trans. Wirel. Commun., vol. 15, no. 6, pp. 44384454, Aug. 2015. DOI: 10.1109/TWC.2016.2542066.

[5] Z. Ding, F. Adachi, and H. V. Poor, "The application of MIMO to non-orthogonal multiple access", IEEE Trans. Wirel. Commun., vol. 15 , no. 1 , pp. 537-552, Sept. 2015. DOI: 10.1109/TWC.2015.2475746.

[6] F. Rusek, D. Persson, B. K. Lau, E. G. Larsson, T. L. Marzetta, O Edfors, and F. Tufvesson, "Scaling up MIMO: Opportunities and challenges with very large arrays", IEEE Signal Process. Mag., vol. 30, no. 1, pp. 40-60, Jan. 2012. DOI: 10.1109/MSP.2011.2178495.

[7] T. L. Marzetta, "How much training is required for multiuser MIMO?", in Proc. of Asilomar Conference on Signals, Systems and Computers, Pacific Grove, CA, USA, 2006, pp. 359-363. DOI: 10.1109/ACSSC.2006.354768

[8] T. E. Bogale and L. B. Le, "Massive MIMO and mmWave for 5G wireless HetNet: Potential benefits and challenges", IEEE Veh. Technol. Mag., vol. 11, no. 1, pp. 64-75, Feb. 2016. DOI: 10.1109/MVT.2015.2496240.

[9] J. Hoydis, S. T. Brink, and M. Debbah, "Massive MIMO in the UL/DL of cellular networks: How many antennas do we need?", IEEE J. Sel. Areas Commun., vol. 31, no. 2, pp. 160-171, Jan. 2013. DOI: 10.1109/JSAC.2013.130205.

[10] A. Gupta and R. K. Jha, "A survey of 5G network: Architecture and emerging technologies", IEEE Access, vol. 3, pp. 1206-1232, Jul. 2015. DOI: 10.1109/ACCESS.2015.2461602.

[11] D. Choudhury, I. Labs, and I. Corporation, "5G wireless and millimeter wave technology evolution: An overview", in Proc. of 2015 International Microwave Symposium, Phoenix, AZ, USA, pp. 0-3. DOI: 10.1109/MWSYM.2015.7167093.

[12] A. J. Paulraj and C. B. Papadias, "Space-time processing for wireless communications", Signal Process. Mag. IEEE, vol. 14, no. 6, pp. 4983, Nov. 1997. DOI: 10.1109/ICASSP.1997.598843.

[13] T. Van Chien, E. Bjornson, and E. G. Larsson, "Downlink power control for massive MIMO cellular systems with optimal user association", in Proc. of 2016 IEEE Int. Conf. Commun., Kuala Lumpur, Malaysia. DOI: 10.1109/ICC.2016.7510950.

[14] J. Jose, A. Ashikhmin, T. L. Marzetta, and S. Vishwanath, "Pilot contamination and precoding in multi-cell TDD systems", IEEE Trans. Wirel. Commun., vol. 10, no. 8, pp. 2640-2651, Jun. 2011. 
DOI: 10.1109/TWC.2011.060711.101155.

[15] L. Lu, G. Y. Li, A. L. Swindlehurst, A. Ashikhmin, and R. Zhang, "An overview of massive MIMO: Benefits and challenges", IEEE Journal on Selected Topics in Signal Processing, vol. 8, no. 5, pp. 742-758, Apr. 2014. DOI: 10.1109/JSTSP.2014.2317671.

[16] S. Biswas, C. Masouros, and T. Ratnarajah, "Performance analysis of large multiuser MIMO systems with space-constrained 2-D antenna arrays", IEEE Trans. Wirel. Commun., vol. 15, no. 5, pp. 3492-3505, Jan. 2016. DOI: 10.1109/TWC.2016.2522419.

[17] S. M. Kay, Fundamentals of Statistical Signal Processing: Estimation Theory. Prentice-Hall Signal Processing Series, 1993, p. 595.

[18] E. Björnson, E. G. Larsson, and M. Debbah, "Massive MIMO for maximal spectral efficiency: How many users and pilots should be allocated?", IEEE Transactions on Wireless Communications, vol.
15, pp. 1293-1308, Oct. 2015. DOI: 10.1109/TWC.2015.2488634.

[19] E. Telatar, "Capacity of multi-antenna Gaussian channels", Eur. Trans. Telecommun., vol. 10 , no. 6, pp. 585-595. DOI: 10.1002/ett.4460100604.

[20] H. Boche and M. Schubert, "A general duality theory for uplink and downlink beamforming", in Proc. of IEEE 56th Veh. Technol. Conf., vol. $1, \quad$ no. $2, \quad 2002$, pp. 87-91. DOI: 10.1109/VETECF.2002.1040308.

[21] T. S. Rappaport, Wireless Communications: Principles and Practice, 2nd ed. Prentice Hall, 2002, pp. 1-736.

[22] V. Saxena, G. Fodor, and E. Karipidis, "Mitigating pilot contamination by pilot reuse and power control schemes for massive MIMO systems", in Proc. of IEEE Vehicular Technology Conference, Glasgow, UK, $2015 . \quad$ DOI 10.1109/VTCSpring.2015.7145932. 